Communications in Physics, Vol. 24, No. 2 (2014), pp. 113-123

\title{
TWO HIGGS SINGLETS A 4 FLAVOR SYMMETRY WITH MINIMAL BREAKING
}

\author{
NGUYEN THANH PHONG \\ Department of Physics, Can Tho University, Can Tho city, Vietnam \\ E-mail: thanhphong@ctu.edu.vn \\ Received 24 April 2014 \\ Accepted for publication 22 June 2014
}

\begin{abstract}
We study the seesaw realization of a $A_{4}$ model with two Higgs singlets. In this model, the mixing angle $\theta_{13}$ and leptogenesis are zero if the components of right handed neutrino mass matrix resulting from the two Higgs singlets are exact degenerate. We then study the minimal breaking of the model by a tiny shift between aforementioned components. This minimal breaking results in deviations of lepton mixing angles from their tri-bimaximal mixing values in which the current experimental value of $\theta_{13}$ can be achieved. Besides, the baryon asymmetry of the Universe is successfully generated through non-zero leptogenesis by the decay of right handed neutrinos.
\end{abstract}

Keywords: $A_{4}$ model, leptogenesis, seesaw mechanism..

\section{INTRODUCTION}

The evidence of neutrino oscillations absolutely confirmed neutrino has mass and they are mixing. Based on neutrino experimental data, in 2002, P. F. Harrison et al. [1] proposed the structure of lepton mixing matrix which named Tri-Bimaximal (TB). According to this structure, the reactor mixing angle, $\theta_{13}$, is zero and the Dirac CP violating phase is meaningless. Subsequently, there were a lot of efforts to find a natural model that leads to TB mixing pattern of leptons, and a fascinating way seems to be the use of some discrete non-Abelian flavor groups added to the gauge groups of the Standard Model (SM). There is a series of models based on the symmetry group $A_{4}$ [2,3], $T^{\prime}$ [4], and $S_{4}$ [5]. The common feature of these models is that they are realized at very high energy scale $\Lambda$ and the groups are spontaneously broken due by a set of scalar multiplets. Based on the latest results of T2K [6], MINOS [7], RENO [8], Double CHOOZ [9] and Daya Bay [10] experiments, the newest values of lepton mixing angles are established where the reactor mixing angle is relatively large [11], $\theta_{13} \sim 8^{0}$. This leads to the necessary of re-evaluating the mentioned models in order to fit with the newest experimental results.

Besides the explaining of lepton mixing pattern, one has to find mechanisms of generating neutrino tinny mass which is absent in SM. And the seesaw mechanism [12] seem to be the most effective one. The seesaw has another appearing feature so-called leptogenesis for the generation of the observed Baryon Asymmetry of the Universe (BAU), through the decay of heavy 
Majorana right handed neutrinos (RHNs) [13]. If the BAU was made via leptogenesis, then $\mathrm{CP}$ violation in the lepton sector is required. For Majorana neutrinos there are one Dirac-type and two Majorana-type $\mathrm{CP}$ violating phases, one (or a combination) of which in principle be measured through neutrinoless double beta $(0 \nu 2 \beta)$ decays [14]. The exact TB mixing pattern forbids at low energy CP violation in neutrino oscillation, due to $U_{e 3}=0$, and also forbids at high energy CP violation in leptogenesis. So any observation of the leptonic $\mathrm{CP}$ violation, for instance in $0 \nu 2 \beta$ decay, can strengthen our believe in leptogenesis by demonstrating that $\mathrm{CP}$ is not a symmetry of the leptons. It is also interesting to explore this existence of $\mathrm{CP}$ violation due to the Majorana CPviolating phases by measuring $\left|\left\langle m_{e e}\right\rangle\right|$ and examine a link between low-energy observable $0 \nu 2 \beta$ decay and the BAU [15].

In this work, we consider the seesaw realization of the $A_{4}$ proposed in [3] which is different with the other $\mathrm{A}_{4}$ models in the reference [2] where there is only one singlet Higgs. In this model, there are two scalar singlets and two scalar triplets in addition to two SM scalar doublets which responsibility for spontaneously breaking of the $A_{4}$ group and the SM gauge group. If the RHN mass matrix's components resulting from the contributions of VEVs of two scalar singlets are the same amount, then the model gives exact TB pattern of lepton mixing matrix and leptogenesis does not work. We then study the case where there is a small shift between the aforementioned entries in the RHN mass matrix which is so called minimal breaking. This tiny difference results in the deviations of lepton mixing angles from their TM values and also successful leptogenesis.

This work is organized as follows. In the next section, Sec. II, we present the overview of the $A_{4}$ model with two Higgs singlets. We discuss the low energy phenomena and leptogenesis of the model in the case in which the RHN mass matrix's components resulting from the contributions of VEVs of two scalar singlets to the RHN mass matrix are the same amount. In Sec. III, we study the case that model is broken minimally. We investigate the low energy obsevables such as the derived reactor mixing angle, neutrinoless double beta decay and the Dirac CP violating phase. The Sec. IV is devoted to calculate leptogenesis and to show our numerical results and discussions. The summary of our work is given in the last section.

\section{OVERVIEW OF THE MODEL}

The non-Abelian $A_{4}$ is a group of even permutations of 4 objects and has $4 ! / 2=12$ elements. The group is generated by two generators $S$ and $T$ satisfying the relations

$$
S^{2}=(S T)^{3}=T^{3}=1 .
$$

There are three one-dimensional irreducible representations of the group denoted as

$$
\begin{aligned}
1: & S=1, T=1 \\
1^{\prime}: & S=1, T=e^{i 4 \pi / 3} \equiv \omega^{2} \\
1^{\prime \prime}: & S=1, T=e^{i 2 \pi / 3} \equiv \omega .
\end{aligned}
$$

The technical details of the group are shown in [16].

In this work, we consider the seesaw realization of the $A_{4}$ model proposed in [3] with two singlet Higgs. In this model, there are four $S U(2)_{L} \otimes U(1)_{L}$ singlet Higgs, two $\left(\xi^{\prime}, \xi^{\prime \prime}\right)$ of which are singlet and the other two $\left(\phi_{S}, \phi_{T}\right)$ transform as triplets under $A_{4}$. The SM lepton doublets are assigned to be the triplet representation of $A_{4}$, while the right handed charged lepton are assumed to belong to the $1,1^{\prime \prime}, 1^{\prime}$ representations, respectively. The standard Higgs doublets $h_{u}$ and $h_{d}$ 
Table 1. List of fermion and scalar fields used in the $A_{4}$ model with two singlet Higgs, $l=e, \mu, \tau$.

\begin{tabular}{lccc}
\hline \hline Lepton & $S U(2)_{L}$ & $A_{4}$ & \\
\hline$\psi^{l}\left(\nu_{l}, l\right)$ & 2 & 3 & \\
$e_{R}$ & 1 & 1 & \\
$\mu_{R}$ & 1 & $1^{\prime \prime}$ & \\
$\tau_{R}$ & 1 & $1^{\prime}$ & \\
$N_{l R}$ & 1 & 3 & \\
\hline Scalar & & & VEV \\
\hline$h_{u}$ & 2 & 1 & $\left\langle h_{u}\right\rangle=v_{u}$ \\
$h_{d}$ & 2 & 1 & $\left\langle h_{d}\right\rangle=v_{d}$ \\
$\phi_{S}$ & 1 & 3 & $\left\langle\phi_{S}\right\rangle=\left(v_{s}, v_{s}, v_{s}\right)$ \\
$\phi_{T}$ & 1 & 3 & $\left\langle\phi_{T}\right\rangle=\left(v_{T}, 0,0\right)$ \\
$\xi^{\prime}$ & 1 & $1^{\prime}$ & $\left\langle\xi^{\prime}\right\rangle=u^{\prime}$ \\
$\xi^{\prime \prime}$ & 1 & $1^{\prime \prime}$ & $\left\langle\xi^{\prime \prime}\right\rangle=u^{\prime \prime}$ \\
\hline \hline
\end{tabular}

remain invariant under $A_{4}$. The particle content for leptons and scalars as well as their VEVs of the considering model is shown in Table 1. The Lagrangian for the lepton sector is given below, here we assume that $\phi_{S}$ does not couple to charged leptons and $\phi_{T}$ does not contributes to the Majorana neutrino mass matrix.

$$
\begin{aligned}
L= & \frac{y_{e}}{\Lambda}\left(\phi_{T} \bar{\psi}_{L}^{l}\right) e_{R} h_{d}+\frac{y_{\mu}}{\Lambda}\left(\phi_{T} \bar{\psi}_{L}^{l}\right)^{\prime} \mu_{R} h_{d}+\frac{y_{\tau}}{\Lambda}\left(\phi_{T} \bar{\psi}_{L}^{l}\right)^{\prime \prime} \tau_{R} h_{d}+f \bar{\psi}_{L}^{l} N_{R} h_{u} \\
& +x_{A}^{\prime} \xi^{\prime}\left(\bar{N}_{L}^{c} N_{R}\right)^{\prime \prime}+x_{A}^{\prime \prime} \xi^{\prime \prime}\left(\bar{N}_{L}^{c} N_{R}\right)^{\prime}+x_{B}\left(\phi_{S} \bar{N}_{L}^{c} N_{R}\right)+h . c .
\end{aligned}
$$

where $\Lambda$ is the cut-off scale of the model. After spontaneous symmetry breaking, the charged lepton mass matrix comes out diagonal with $m_{e}=\frac{y_{e} v_{T} v_{d}}{\Lambda}, m_{\mu}=\frac{y_{\mu} v_{T} v_{d}}{\Lambda}$, and $m_{\tau}=\frac{y_{\tau} v_{T} v_{d}}{\Lambda}$. The neutrino sector gives rise to the following Dirac and Majorana neutrino mass matrices

$$
m_{D}=f v_{u}\left(\begin{array}{lll}
1 & 0 & 0 \\
0 & 1 & 0 \\
0 & 0 & 1
\end{array}\right), M_{R}=\left(\begin{array}{ccc}
\frac{2 B}{3} & C-\frac{B}{3} & D-\frac{B}{3} \\
C-\frac{B}{3} & D+\frac{2 B}{3} & -\frac{B}{3} \\
D-\frac{B}{3} & -\frac{B}{3} & C+\frac{2 B}{3}
\end{array}\right),
$$

where $B=2 x_{B} v_{S}, D=2 x_{A}^{\prime} u^{\prime}, C=2 x_{A}^{\prime \prime} u^{\prime \prime}$. If $C=D$, the Majorana neutrino mass matrix is diagonalized by TB mixing matrix given in Eq. (8) and the eigenvalues of RHN masses are $M_{1}=B-C, M_{2}=2 C, M_{3}=B+C$. The structure of light neutrino mass matrix can be obtained from see-saw formula [12]:

$$
m_{\nu}=m_{D} M_{R}^{-1} m_{D}^{T}=U_{T B}\left(\begin{array}{ccc}
\frac{f^{2} v_{u}^{2}}{B-C} & 0 & 0 \\
0 & \frac{f^{2} v_{u}^{2}}{2 C} & 0 \\
0 & 0 & \frac{f^{2} v_{u}^{2}}{B+C}
\end{array}\right) U_{T B}^{T},
$$


where

$$
U_{T B}=\left(\begin{array}{ccc}
\frac{\sqrt{2}}{\sqrt{3}} & \frac{1}{\sqrt{3}} & 0 \\
-\frac{1}{\sqrt{6}} & \frac{1}{\sqrt{3}} & -\frac{1}{\sqrt{2}} \\
-\frac{1}{\sqrt{6}} & \frac{1}{\sqrt{3}} & \frac{1}{\sqrt{2}}
\end{array}\right) .
$$

It is clear from Eq. (7) that $U_{\mathrm{TB}}$ is the diagonalizing matrix for the light neutrino mass matrix $m_{\nu}$ as well. From Eqs. $(7,8)$, we derive the eigenvalues of $m_{\nu}$ and the mixing angles as

$$
\begin{gathered}
m_{1}=\frac{f^{2} v_{u}^{2}}{B-C}, m_{2}=\frac{f^{2} v_{u}^{2}}{2 C}, m_{3}=\frac{f^{2} v_{u}^{2}}{B+C}, \\
\sin \theta_{12}=1 / \sqrt{3}, \sin \theta_{23}=-1 / \sqrt{2}, \sin \theta_{13}=0 .
\end{gathered}
$$

And from Eq. (9) we get the solar and atmospheric mass-squared differences as

$$
\Delta m_{\odot}^{2}=m_{2}^{2}-m_{1}^{2}=m_{0}^{2} \frac{k^{2}-2 k-3}{(k-1)^{2}}, \Delta m_{\mathrm{atm}}^{2}=m_{3}^{2}-m_{2}^{2}=m_{0}^{2} \frac{3-2 k-k^{2}}{(k+1)^{2}},
$$

where $M_{0}=2 C, B=k C, m_{0}=\frac{f^{2} v_{u}^{2}}{M_{0}}$ and all the parameter are real. From the experiments we know $\Delta m_{\odot}^{2}$ is positive and dictates either $k<-1$ or $k>3$. If $k<-1$, it is required that $|k+1|$ should be small in order to generate a small value of $\Delta m_{\odot}^{2}$ provided that $m_{0}^{2}$ is not too small as $\Delta m_{\odot}^{2}$. And if it is the case, the hierarchy of $\Delta m_{\odot}^{2}$ and $\Delta m_{\text {atm }}^{2}$ is obtained with the singularity of $\Delta m_{\text {atm }}^{2}$ near $k \simeq-1$. If $|k|$ increases, we can get $m_{0}^{2} \simeq \Delta m_{\odot}^{2}$ but it lead to the same order of magnitude of $\Delta m_{\odot}^{2}$ and $\Delta m_{\text {atm }}^{2}$ which is not acceptable according to experimental results. And this corresponds to the normal hierarchical mass spectrum. Now, for $m_{0}^{2} \gg \Delta m_{\odot}^{2}$ $\left(m_{0}^{2} \simeq \Delta m_{\mathrm{atm}}^{2}\right), k>3$ is the physical region. This region makes $\Delta m_{\mathrm{atm}}^{2}<0$ which is so-called inverted hierarchy of the light neutrino masses. Again, $(k-3)$ has to be small in order to generate a small value of $\Delta m_{\odot}^{2}$.

For one complex parameter $C \equiv C e^{i \phi}$, the mass differences are obtained as

$$
\Delta m_{\odot}^{2}=m_{2}^{2}-m_{1}^{2}=m_{0}^{2} \frac{k^{2}-2 k \cos \phi-3}{1+k^{2}-2 k \cos \phi}, \Delta m_{\mathrm{atm}}^{2}=m_{3}^{2}-m_{2}^{2}=m_{0}^{2} \frac{3-2 k \cos \phi-k^{2}}{1+k^{2}+2 k \cos \phi} .
$$

In the complex case, the positivity of $\Delta m_{\odot}^{2}$ can be obtained either with $k<\left(\cos \phi-\sqrt{3+\cos ^{2} \phi}\right)$ or with $k>\left(\cos \phi+\sqrt{3+\cos ^{2} \phi}\right)$. For the first case with $m_{0}^{2} \simeq \Delta m_{\odot}^{2}$ and with $\cos \phi \simeq \frac{-1-k^{2}}{2 k}$ one can have normal hierarchical mass spectrum. For the second case hierarchy will be inverted and $\left(k>\cos \phi+\sqrt{3+\cos ^{2} \phi}\right)$ has to be small. In both cases $k$ should take the value such that the $1 \geq \cos \phi \geq-1$ range also satisfies. The mixing pattern is the TB in Eq. (8) and it is independent of whether the parameters are real or complex. In this mixing pattern $U_{e 3}=0$ and notice that nonzero complex $U_{e 3}$ is a basic requirement to see the nonzero Dirac CP violation.

Now we concentrate on the issue of leptogenesis of this model. The decay of RHNs to a lepton (charged or neutral) and scalar (charged or neutral) generate nonzero lepton asymmetry if (i) $C$ and $C P$ are violated, (ii) the lepton number is violated, and (iii) the decay of RHNs are out of equilibrium. We are in the energy scale where $A_{4}$ symmetry is broken but the SM gauge 
group remains unbroken, so the Higgs scalars, both charged and neutral, are physical. The CP asymmetry of the decay is characterized by a parameter $\varepsilon_{i}$ which is defined as

$$
\varepsilon_{i}=\frac{\Gamma\left(N_{i} \rightarrow l \varphi\right)-\Gamma\left(N_{i} \rightarrow \bar{l} \varphi^{\dagger}\right)}{\Gamma\left(N_{i} \rightarrow l \varphi\right)+\Gamma\left(N_{i} \rightarrow \bar{l} \varphi^{\dagger}\right)}
$$

Spontaneous $A_{4}$ symmetry breaking generates the RHN mass and the mass matrix $M_{R}$ obtained is shown in Eq. (6). We need to diagonalize $M_{R}$ in order to go into the physical basis (mass basis) of RHN.

$$
U_{T B}^{T} M_{R} U_{T B}=\operatorname{diag}\left(M_{1}, M_{2}, M_{3}\right)=\operatorname{diag}(B-C, 2 C, B+C),
$$

however, the eigenvalues are not real. We need to multiply one diagonal phase matrix $U_{P}$ with $U_{T B}$. Hence, diagonalizing the matrix $V=U_{T B} U_{P}$ relates the flavor basis to the eigenbasis of the right-handed neutrino:

$$
N_{l R}=\sum_{i=1}^{3} V_{l i} N_{i R}
$$

In this basis the couplings of $N_{R}$ with leptons and scalars are modified and it will be

$$
m_{D}^{\prime}=V^{T} m_{D}
$$

At the tree level there is no asymmetry in the decay of RHNs. Because of the interference between tree-level and one-loop level diagrams, the asymmetry is generated. The $\mathrm{CP}$ asymmetry generated through the interference between tree and one-loop diagrams for the decay of the RHN $N_{i}$ is given [17]

$$
\varepsilon_{i}=\frac{1}{8 \pi v_{u}^{2} H_{i i}} \sum_{j \neq i} \operatorname{Im}\left[H_{i j}^{2}\right] g\left(\frac{M_{j}^{2}}{M_{i}^{2}}\right),
$$

where $H$ is the Hermitian matrix defined as $H=m_{D}^{\prime} m_{D}^{\prime \dagger}$ and $g(x)$ is given by

$$
g(x)=\sqrt{x}\left[\frac{1}{1-x}+1-(1+x) \ln \frac{1+x}{x}\right] .
$$

The key matrix, whose elements are necessary to calculate leptogenesis, is $H$. In this model, we find that the $H$ matrix is real diagonal and proportional to the identity matrix. Therefore, the decay of all three generations of RHNs could not generate lepton asymmetry. As a result, this model of $A_{4}$ symmetry is not compatible with the low energy Dirac CP violation, reactor mixing angle as well as with high energy $\mathrm{CP}$ violation. In order to obtain nonzero $\theta_{13}$, low energy Dirac $\mathrm{CP}$ violation, and leptogenesis we need to break the $A_{4}$ symmetry through not only spontaneously, but also explicitly, introducing some soft $A_{4}$ symmetry breaking [15] terms in the Lagrangian and/or considering the group evolution effects [18]. In this work, we consider the minimal breaking exact $A_{4}$ symmetry which by considering a tiny hierarchy between the $C$ and $D$ parameters of RNH mass matrix. 


\section{TWO SINGLET HIGGS $A_{4}$ WITH MINIMAL SYMMETRY BREAKING}

In this section, we consider the minimal breaking of $A_{4}$ symmetry through a tiny hierarchy between the components of $M_{R}$ coming from the VEVs of $\xi^{\prime}$ and $\xi^{\prime \prime},\left(C=D\left(e^{i \phi}+2 \rho\right)\right)$ while keeping $m_{D}$ unchanged. The RHN mass matrix now is modified as

$$
M_{R}=\left(\begin{array}{ccc}
\frac{2 B}{3} & D\left(e^{i \phi}+2 \rho\right)-\frac{B}{3} & D e^{i \phi}-\frac{B}{3} \\
D\left(e^{i \phi}+2 \rho\right)-\frac{B}{3} & D e^{i \phi}+\frac{2 B}{3} & \frac{B}{3} \\
D e^{i \phi}-\frac{B}{3} & -\frac{B}{3} & D\left(e^{i \phi}+2 \rho\right)+\frac{2 B}{3}
\end{array}\right),
$$

where we assume $C$ and $D$ to be complex in order to associate with leptogenesis and other CP violating processes. The mass matrix $M_{R}$ is diagonalized by a modified mixing matrix $\tilde{U}_{T B}$ as

$$
\begin{aligned}
\tilde{U}_{T B}^{T} M_{R} \tilde{U}_{T B} & =\operatorname{diag}\left(M_{1}, M_{2}, M_{3}\right) \\
& =\operatorname{diag}\left(B-D\left(e^{i \phi}+\rho\right), 2 D\left(e^{i \phi}+\rho\right), B+D\left(e^{i \phi}+\rho\right)\right),
\end{aligned}
$$

where up to the first order of $\rho$, the $\tilde{U}_{T B}$ matrix a obtained as

$$
\tilde{U}_{T B}=\left(\begin{array}{ccc}
\frac{\sqrt{2}}{\sqrt{3}} & \frac{1}{\sqrt{3}} & -\frac{\rho}{\sqrt{2}} e^{-i \phi} \\
-\frac{1}{\sqrt{6}}-\frac{\sqrt{3}}{2 \sqrt{2}} \rho e^{-i \phi} & \frac{1}{\sqrt{3}} & -\frac{1}{\sqrt{2}}+\frac{\rho}{2 \sqrt{2}} e^{-i \phi} \\
-\frac{1}{\sqrt{6}}+\frac{\sqrt{3}}{2 \sqrt{2}} \rho e^{-i \phi} & \frac{1}{\sqrt{3}} & \frac{1}{\sqrt{2}}+\frac{\rho}{2 \sqrt{2}} e^{-i \phi}
\end{array}\right) .
$$

The structure of light neutrino mass matrix is given through the seesaw as

$$
m_{\nu}=m_{D} M_{R}^{-1} m_{D}^{T}=\tilde{U}_{T B}\left(\begin{array}{ccc}
\frac{f^{2} v_{u}^{2}}{B-D\left(e^{i \phi}+\rho\right)} & 0 & 0 \\
0 & \frac{f^{2} v_{u}^{2}}{2 D\left(e^{i \phi}+\rho\right)} & 0 \\
0 & 0 & \frac{f^{2} v_{u}^{2}}{B+D\left(e^{i \phi}+\rho\right)}
\end{array}\right) \tilde{U}_{T B}^{T} .
$$

From this equation we obtain the light neutrino mass eigenvalues

$$
m_{1}=\frac{f^{2} v_{u}^{2}}{B-D\left(e^{i \phi}+\rho\right)}, m_{2}=\frac{f^{2} v_{u}^{2}}{2 D\left(e^{i \phi}+\rho\right)}, m_{3}=\frac{f^{2} v_{u}^{2}}{B+D\left(e^{i \phi}+\rho\right)},
$$

and hence we obtain

$$
\begin{aligned}
& m_{1}^{2}=\frac{4 m_{0}^{2}}{1+(k-\rho)^{2}-2(k-\rho) \cos \phi}, m_{2}^{2}=\frac{m_{0}^{2}}{1+\rho^{2}+2 \rho \cos \phi}, \\
& m_{3}^{2}=\frac{4 m_{0}^{2}}{1+(k+\rho)^{2}+2(k+\rho) \cos \phi},
\end{aligned}
$$

where $m_{0}, k$ are defined as before. Then we can obtain the squared differences

$$
\Delta m_{\odot}^{2}=m_{2}^{2}-m_{1}^{2}, \Delta m_{\mathrm{atm}}^{2}=m_{3}^{2}-m_{2}^{2} .
$$

In Fig. 1 we present the allowed parameter space $(k, \rho, \phi)$ constrained by the experimental data given in [11] at $3 \sigma$ level. Thereafter we have used $m_{0}^{2} \simeq \Delta m_{\text {sol }}^{2}$ (best fit) and $M_{0}=10^{12} \mathrm{GeV}$ as universal input. The light neutrino masses are inverted hierarchy $\left(m_{2}>m_{1} \gg m_{3}\right)$.

From the mixing matrix $\tilde{U}_{T B}$, the deviation of the mixing angles from TB are obtained as

$$
D_{12} \simeq 0, D_{23} \simeq-\frac{\rho}{2}, U_{13}=-\frac{\rho e^{-i \phi}}{\sqrt{2}},
$$


where $D_{12}=\sin ^{2} \theta_{12}-1 / 3$ and $D_{23}=\sin ^{2} \theta_{23}-1 / 2$. We can see that, the current value of $\theta_{13}$ can be achieved by a suitable choice of the braking parameter $\rho$. However, the value of $\rho$ is also constrained by the value of angle $\theta_{23}$.
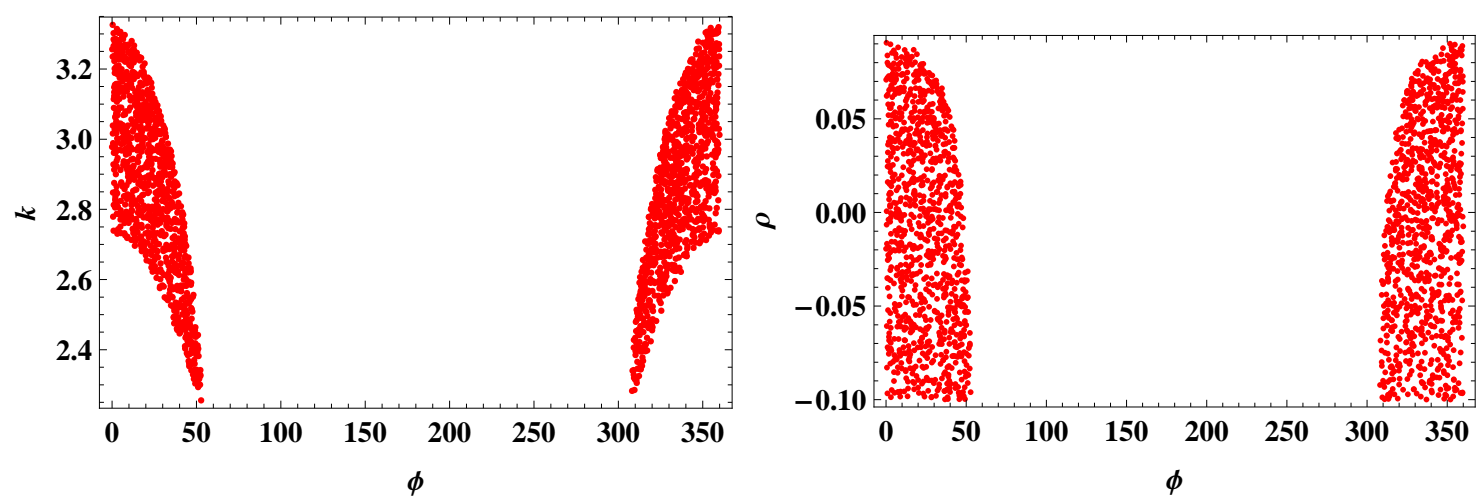

Fig. 1. The allowed parameter space of the model constrained by the experimental data given in [11]. We have used $m_{0}^{2} \simeq \Delta m_{\mathrm{sol}}^{2}$ (best fit) and $M_{0}=10^{12} \mathrm{GeV}$ as universal input. The light neutrino masses are inverted hierarchy $\left(m_{2}>m_{1} \gg m_{3}\right)$.
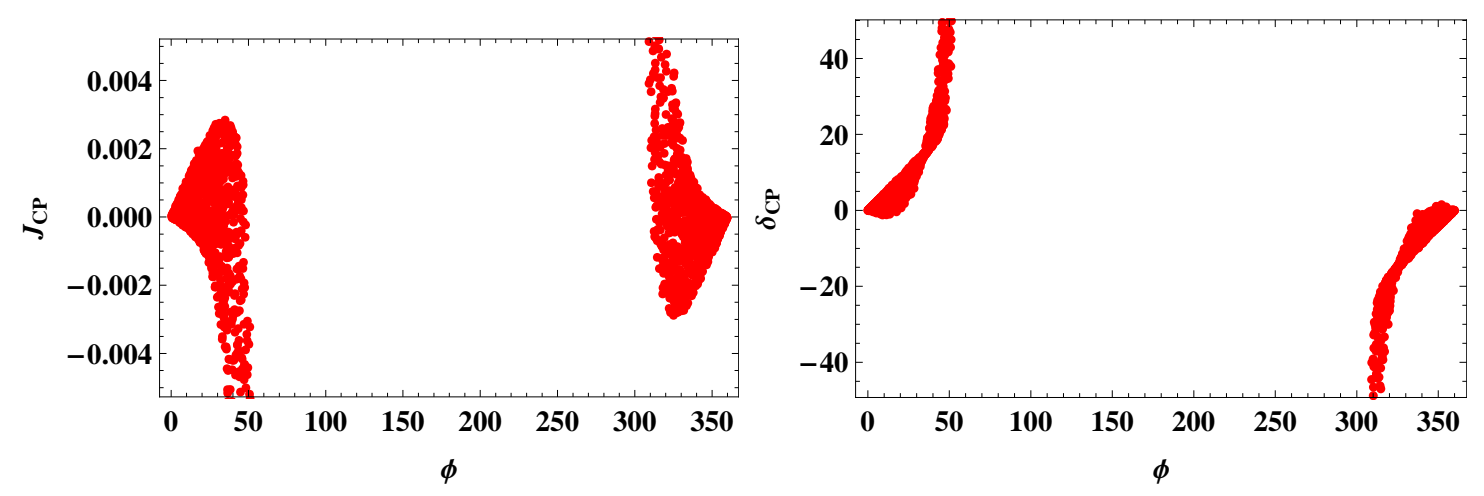

Fig. 2. Predictions of the Jarlskog invariant parameter $J_{\mathrm{CP}}$ (left panel) and Dirac CP violating phase $\delta_{\mathrm{CP}}$ (right panel) as a function of $\phi$.

Another important point here is that, because the exist of a complex parameter in the RHN mass matrix, we can extract the Dirac CP violation phase $\delta_{\mathrm{CP}}$ from the Jarlskog invariant [19] given by

$$
\begin{aligned}
J_{\mathrm{CP}} & =\frac{1}{8} \sin 2 \theta_{12} \sin 2 \theta_{23} \sin 2 \theta_{13} \cos \theta_{13} \sin \delta_{\mathrm{CP}} \\
& =\frac{\operatorname{Im}\left[h_{12} h_{23} h_{31}\right]}{\Delta m_{21}^{2} \Delta m_{31}^{2} \Delta m_{32}^{2}}
\end{aligned}
$$


where $h=m_{\nu}^{\dagger} m_{\nu}$ and up to the first order of $\rho$ we get

$$
\begin{aligned}
h_{12}= & \frac{m_{0}^{2}}{3\left(1+k^{4}-2 k^{2} c_{2 \phi}\right)} \\
& \times \frac{k^{4}-k^{2}\left(4+2 c_{2 \phi}+12 \rho c_{\phi}\right)-8 k\left[c_{\phi}+\rho\left(5+c_{2 \phi}\right)\right]-3\left(1+4 \rho c_{\phi}-i 8 \rho s_{\phi}\right)}{1+2 \rho c_{\phi}+\frac{4 \rho\left(1-k^{2}\right) c_{\phi}}{1+k^{4}-2 k^{2} c_{2 \phi}}}, \\
h_{23}= & \frac{m_{0}^{2}}{3\left(1+k^{4}-2 k^{2} c_{2 \phi}\right)} \\
& \times \frac{k^{4}-k^{2}\left(4+2 c_{2 \phi}+12 \rho c_{\phi}\right)+16 k\left[c_{\phi}+\rho\left(2+c_{2 \phi}\right)\right]-3\left(1+4 \rho c_{\phi}-i 8 \rho s_{\phi}\right)}{1+2 \rho c_{\phi}+\frac{4 \rho\left(1-k^{2}\right) c_{\phi}}{1+k^{4}-2 k^{2} c_{2 \phi}}}, \\
h_{12}= & \frac{m_{0}^{2}}{3\left(1+k^{4}-2 k^{2} c_{2 \phi}\right)} \quad 1+2 \rho c_{\phi}+\frac{4 \rho\left(1-k^{2}\right) c_{\phi}}{1+k^{4}-2 k^{2} c_{2 \phi}} \\
& \times \frac{k^{4}-k^{2}\left(4+2 c_{2 \phi}+12 \rho c_{\phi}\right)-8 k\left[c_{\phi}-\rho\left(1-c_{2 \phi}\right)\right]-3\left(1+4 \rho c_{\phi}-i 8 \rho s_{\phi}\right)}{1+28},
\end{aligned}
$$

where $c_{\phi}=\cos \phi, s_{\phi}=\sin \phi$ and $c_{2 \phi}=\cos 2 \phi$. The predictions of the Jarlskog invariant parameter $J_{\mathrm{CP}}$ (left panel) and Dirac CP violating phase $\delta_{\mathrm{CP}}$ (right panel) as a function of $\phi$ are plotted in Fig. 2.

Now let us consider the neutrinoless double beta decay which is related with the absolute value of the ee-element of light neutrino mass matrix and is, up to the first order of $\rho$, approximately given in our scenario by

$$
\begin{aligned}
\left|\left\langle m_{e e}\right\rangle\right| & =\left|\frac{m_{0}}{3} \times\left(\frac{1}{\rho+e^{i \phi}}+\frac{4\left(k+\rho+e^{i \phi}\right)}{k^{2}-2 \rho e^{i \phi}-e^{i 2 \phi}}\right)\right| \\
& =\frac{m_{0}^{2}\left[k^{4}+8 k^{3}\left(\rho+2 c_{\phi}\right)+k^{2}\left(16+76 \rho c_{\phi}+6 c_{2 \phi}\right)+24 k\left(2 \rho+2 c_{\phi}+\rho c_{2 \phi}\right)+9\left(1+4 \rho c_{\phi}\right)\right]}{3\left(1+k^{4}-2 k^{2} c_{2 \phi}\right)\left(1+2 \rho c_{\phi}+\frac{4 \rho c_{\phi}\left(1-k^{2}\right)}{1+k^{4}-2 k^{2} c_{\phi}}\right)},
\end{aligned}
$$

The prediction of $0 \nu \beta \beta$ parameter, $\left|\left\langle m_{e e}\right\rangle\right|$, is shown in the left panel of Fig. 3 as a function of $\phi$. This prediction is below the current lower bound sensitivity $(0.2 \mathrm{eV})[20]$ and above the future below lower bound sensitivity $\left(10^{-2} \mathrm{eV}\right)[21]$

\section{LEPTOGENESIS IN THE $A_{4}$ MODEL WITH MINIMAL SYMMETRY BREAKING}

This section is devoted to consider how leptogenesis can work in our scenario. First we diagonalize the RNH mass matrix $M_{R}$ as

$$
V^{T} M_{R} V=\operatorname{diag}\left(M_{1}, M_{2}, M_{3}\right),
$$

where

$$
V=\tilde{U}_{T B} \times U_{P}=\tilde{U}_{T B} \times\left(\begin{array}{ccc}
e^{i \alpha} & 0 & 0 \\
0 & e^{i \beta} & 0 \\
0 & 0 & e^{i \gamma}
\end{array}\right)
$$




$$
\begin{aligned}
\tan 2 \alpha & =\frac{\sin \phi}{\rho-k+\cos \phi}, \tan 2 \beta=\frac{\sin \phi}{\rho+\cos \phi}, \tan 2 \gamma=\frac{\sin \phi}{\rho+k+\cos \phi}, \\
\tilde{U}_{T B} & =\left(\begin{array}{ccc}
\frac{\sqrt{2}}{\sqrt{3}} & \frac{1}{\sqrt{3}} & -\frac{\rho}{\sqrt{2}} e^{-i \phi} \\
-\frac{1}{\sqrt{6}}-\frac{\sqrt{3}}{2 \sqrt{2}} \rho e^{-i \phi} & \frac{1}{\sqrt{3}} & -\frac{1}{\sqrt{2}}+\frac{\rho}{2 \sqrt{2}} e^{-i \phi} \\
-\frac{1}{\sqrt{6}}+\frac{\sqrt{3}}{2 \sqrt{2}} \rho e^{-i \phi} & \frac{1}{\sqrt{3}} & \frac{1}{\sqrt{2}}+\frac{\rho}{2 \sqrt{2}} e^{-i \phi}
\end{array}\right)
\end{aligned}
$$

Then, the Dirac neutrino mass matrix in the new basis is transformed as

$$
m_{D}^{\prime}=V^{T} m_{D}=f v_{u}\left(\begin{array}{ccc}
\frac{\sqrt{2}}{\sqrt{3}} e^{-i \alpha} & \frac{1}{\sqrt{3}} e^{-i \beta} & -\frac{\rho}{\sqrt{2}} e^{i(\phi-\gamma)} \\
-\frac{1}{2 \sqrt{6}} e^{-i \alpha}\left(2+3 \rho e^{i \phi}\right) & \frac{1}{\sqrt{3}} e^{-i \beta} & \frac{1}{2 \sqrt{2}} e^{-i \gamma}\left(-2+\rho e^{i \phi}\right) \\
-\frac{1}{2 \sqrt{6}} e^{-i \alpha}\left(-2+3 \rho e^{i \phi}\right) & \frac{1}{\sqrt{3}} e^{-i \beta} & \frac{1}{2 \sqrt{2}} e^{-i \gamma}\left(2+\rho e^{i \phi}\right)
\end{array}\right)
$$

Leading to the Hermitian matrix $H$ which is relevant to leptogenesis is obtained as

$$
H=m_{D}^{\prime} m_{D}^{\prime \dagger}=f^{2} v_{u}^{2}\left(\begin{array}{ccc}
1+\rho^{2} / 2 & i \rho \sin \phi-\rho^{2} / 4 & -i \rho \sin \phi-\rho^{2} / 4 \\
-i \rho \sin \phi-\rho^{2} / 4 & 1+\rho^{2} / 2 & i \rho \sin \phi-\rho^{2} / 4 \\
i \rho \sin \phi-\rho^{2} / 4 & -i \rho \sin \phi-\rho^{2} / 4 & 1+\rho^{2} / 2
\end{array}\right)
$$

We can see that the off-diagonal terms of the $H$ matrix are all complex, then the CP asymmetry, $\varepsilon_{i}$, is generated by the decay of the RHN $N_{i}$, see Eq. (17).
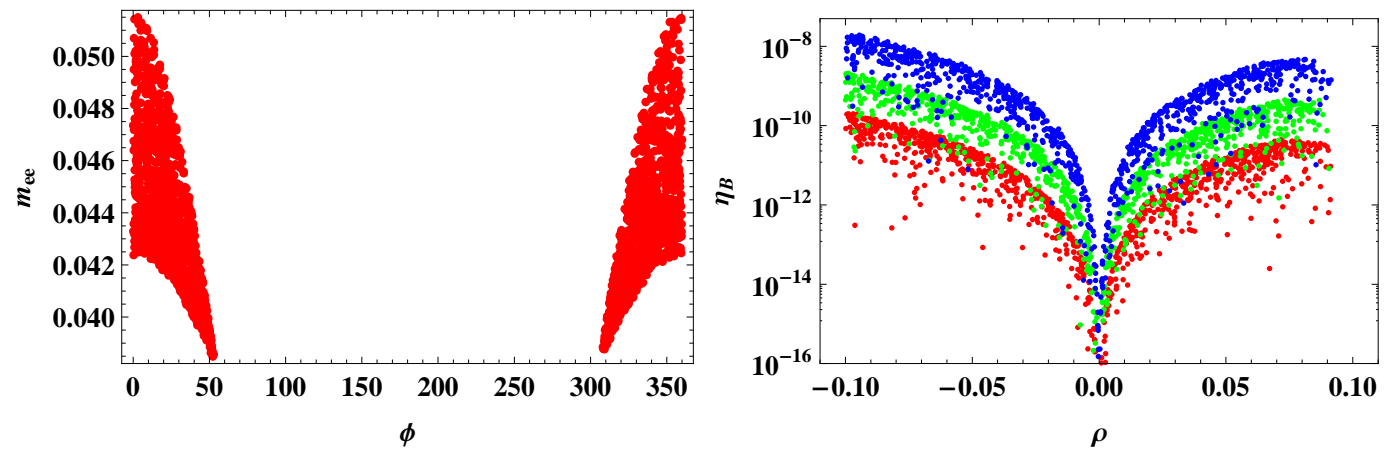

Fig. 3. Predictions of the effective mass $\left|\left\langle m_{e e}\right\rangle\right|$ for $0 \nu 2 \beta$ as a function of $\phi$ in the left panel. The right panel shows the prediction of BAU, $\eta_{B}$, as a function of minimal breaking parameter $\rho$. The red, green and blue patterns respectively correspond to three scales of RHN mass, $M_{0}=10^{11}, 10^{12}, 10^{13} \mathrm{GeV}$.

In addition to $\varepsilon_{i}$, in the conventional leptogenesis, it is well know that the baryon asymmetry also depends on the parameters, which are called washout effect:

$$
K_{i} \equiv \frac{\Gamma_{i}}{H}=\frac{\tilde{m}_{i}}{m_{*}}
$$

where $\Gamma_{i}$ is the three level decay width of $N_{i}$ and $H$ is the Hubble constant. Here the effective neutrino mass, $\tilde{m}_{i}$, a measure of the strength of the coupling of $N_{i}$ to the thermal bath is defined as

$$
\tilde{m}_{i}=\frac{\left[m_{D}^{\prime} m_{D}^{\prime \dagger}\right]_{i i}}{M_{i}}
$$


and $m_{*}$ is the so-call equilibrium neutrino mass and defined as

$$
m_{*}=\frac{16 \pi^{5 / 2}}{3 \sqrt{5}} g_{*}^{1 / 2} \frac{v_{u}^{2}}{M_{\text {Planck }}} \simeq 10^{-3} \mathrm{eV},
$$

where we adopted $M_{\text {Planck }}=1.22 \times 10^{19} \mathrm{GeV}$. And the effective number degrees of freedom in SM with three right-handed neutrinos and one extra Higgs doublet is 116. After reprocessing by sphaleron transitions, the baryon asymmetry is related to $(B-L)$ asymmetry by $Y_{B}=$ $(8 / 23) Y_{B-L}$ [22]. In the conventional letogenesis, we are always in the strong washout regime with $K_{i}>1$ and the RHN $N_{i}^{\prime}$ s are nearly in thermal equilibrium. Then, the generated $B-L$ asymmetry in the strong washout regime is given by [23]

$$
Y_{B-L} \simeq \sum_{i} 0.3 \frac{\varepsilon_{i}}{g_{*}}\left(\frac{0.55 \times 10^{-3} \mathrm{eV}}{\widetilde{m}_{i}}\right)^{1.16}
$$

where we have take into account the contributions of all three generations of RHN neutrinos since their masses are almost degenerate. Then, the resulting baryon-to-photon ration becomes,

$$
\eta_{B}=\left[\frac{s}{n_{\gamma}}\right]_{0} \cdot \frac{n_{B}}{s} \simeq 7.04 Y_{B} \simeq \frac{17}{23} \sum_{i} \frac{\varepsilon_{i}}{g_{*}}\left(\frac{0.55 \times 10^{-3} \mathrm{eV}}{\tilde{m}_{i}}\right)^{1.16},
$$

where the zero indicates the present time. The prediction of $\eta_{B}$ as a function of minimal breaking parameter $\rho$ is shown in the right panel of Fig. 3. The red, green and blue patterns respectively correspond to three scales of RHN mass, $M_{0}=10^{11}, 10^{12}, 10^{13} \mathrm{GeV}$. At the present, the experimental value of baryon asymmetry given in the reference [24] is about $\eta_{B}^{\mathrm{CMB}}=6.225 \times 10^{-10}$,

and the phenomenologically allowed regions is about $2 \times 10^{-10} \leq \eta_{B} \leq 10^{-9}$. Therefore, the mass scale of RHN in our scenario is required about $10^{12} \mathrm{GeV}$ for successful leptogenesis.

\section{SUMMARY}

We have considered the seesaw model of $A_{4}$ symmetry with two Higgs singlets. It is shown that, if the components of RHN mass matrix resulting from VEVs of the two Higgs singlets are degenerate, then the lepton mixing matrix has the TB structure. According to the TB pattern, the reactor mixing angle, $\theta_{13}$, is zero which is disagreed with the current neutrino experimental data. Besides, there is no $\mathrm{CP}$ violation by the decay of RHNs leading to the BAU is could not explained by the model. We then considered a tiny shift between the aforementioned components of RHN mass matrix. This tiny minimal breaking parameter leads to deviations of lepton mixing angles from their TB values. As a results, the current value of $\theta_{13}$ can be achieved by the model by choosing a suitable value of breaking parameter, $\rho$. An other interesting result of minimal breaking of the model is that the BAU is successfully generated by the decay of RHNs. We also investigated the Jarlskog parameter, $J_{\mathrm{CP}}$, which is an invariant CP violation parameter in neutrino oscillations. The neutrinoless double beta decay parameter $\left(\left|\left\langle m_{e e}\right\rangle\right|\right)$ and the Dirac CP violating phase $\left(\delta_{\mathrm{CP}}\right)$ are also studied in this letter.

Notice that, this work studies the case of conventional leptogenesis where the flavor effects are not taken into account. If we consider the flavored leptogenesis where the effects of lepton flavors are included, the the mass scale of RHN for successful leptogenesis is much lower. Besides, in this work, the numerical calculation is for the case of inverted hierarchy of neutrino mass, the case of normal hierarchy can be calculated similarly. 


\section{ACKNOWLEDGMENTS}

This work is funded by the Vietnam National Foundation for Science and Technology Development (NAFOSTED).

\section{REFERENCES}

[1] P. F. Harrison, D. H. Perkins, W. G. Scott, Phys. Lett. B 530 (2002) 167 [arXiv:hep-ph/0202074]. P. F. Harrison, W. G. Scott, Phys. Lett. B 535 (2002) 163 [arXiv:hep-ph/0203209]. P. F. Harrison, W. G. Scott, Phys. Lett. B 547 (2002) 219. P. F. Harrison, W. G. Scott, Phys. Lett. B 557 (2003) 76.

[2] E. Ma and G. Rajasekaran, Phys. Rev. D 64 (2001) 113012 [arXiv:hep-ph/0106291]; K. S. Babu, E. Ma and J. W. F. Valle, Phys. Lett. B 552 (2003) 207 [arXiv:hepph/0206292]; G. Altarelli and F. Feruglio, Nucl. Phys. B 720 (2005) 64 [arXiv:hep-ph/0504165]; Nucl. Phys. B 741, (2006) 215; F. Bazzocchi, S. Kaneko and S. Morisi, JHEP 0803 (2008) 063 [arXiv:0707.3032 [hepph]].

[3] Biswajoy Brahmachari et al. Phys. Rev. D 77 (2008) 073008.

[4] F. Feruglio, C. Hagedorn, Y. Lin and L. Merlo, Nucl. Phys. B 775 (2007) 120 [arXiv:hep-ph/0702194]; M. C. Chen and K. T. Mahanthappa, Phys. Lett. B 652 (2007) 34 [arXiv:0705.0714 [hep-ph]]; P. H. Frampton and T. W. Kephart, JHEP 0709 (2007) 110 [arXiv:0706.1186 [hep-ph]]; P. H. Frampton and S. Matsuzaki, arXiv:0902.1140 [hep-ph].

[5] S. Pakvasa and H. Sugawara, Phys. Lett. B 82 (1979) 105; T. Brown, N. Deshpande, S. Pakvasa and H. Sugawara, Phys. Lett. B 141 (1984) 95; D. G. Lee and R. N. Mohapatra, Phys. Lett. B 329 (1994) 463 [arXiv:hepph/9403201]; E. Ma, Phys. Lett. B 632 (2006) 352 [arXiv:hep-ph/0508231];

[6] T2K Collaboration, K. Abe et al., Phys. Rew. Lett. 107 (2011) 041801

[7] MINOS Collaboration, P. Adamson et al., Phys. Rew. Lett. 107 (2011) 181802

[8] RENO Collaboration, J. Ahn et al., Phys. Rev. Lett. 108 (2012) 191802 [arXiv:hep-ex/1204.0626].

[9] Double CHOOZE Collaboration, Y. Abe et al., Phys. Rew. Lett. 108 (2012) 231081.

[10] Daya Bay Collaboration, F. An et al., Phys. Rew. Lett. 108 (2012) 171803.

[11] D.V. Forero et al., Phys.Rev. D 86 (2012) 073012 [arXiv:hep-ph/1205.4018].

[12] P. Minkowski, Phys. Lett. B 67 (1977) 421; R. N. Mohapatra, G. Senjanovic, Phys. Rev. Lett. 44, (1980) 912.

[13] M. Fukugita and T. Yanagida, Phys. Lett. B 174 (1986) 45; G. F. Giudice et al., Nucl. Phys. B 685 (2004) 89 [arXiv:hep-ph/0310123]; W. Buchmuller, P. Di Bari and M. Plumacher, Annals Phys. 315 (2005) 305 [arXiv:hep$\mathrm{ph} / 0401240]$

[14] S. M. Bilenky, S. Pascoli, and S. T. Petcov, Phys. Rev. D 64 (2001) 053010; S. Pascoli, S. T. Petcov, and L. Wolfenstein, Phys. Lett. B 524 (2002) 319; 549 (2002) 177; S. T. Petcov, New J. Phys. 6 (2004) 109.

[15] Y.H. Ahn, S. K. Kang, C.S. Kim, T.Phong Nguyen, Phys. Rev. D 82 (2010) 093005.

[16] Nguyen Thanh Phong, Communications in Physics 24 (2014) 9-19.

[17] T. Endoh, T. Morozumi, Z. Xiong, Prog. Theor. Phys. 111123 (2004) [arXive:hep-ph/0308276]; A. Abada, S. Davidson, A. Ibarra, F. X. Josse-Michaux, M. Losada and A. Riotto, JHEP 0609, (2006) 010 [arXiv:hep$\mathrm{ph} / 0605281]$

[18] T. Phong Nguyen, P. V. Dong, Adv. High Energy Phys. 2012 (2012) 254093.

[19] C. Jarlskog, Phys. Rev. Lett. 55, (1985) 1039; D. D. Wu, Phys. Rev. D 33 (1986) 860.

[20] H.V. Klapdor-Kleingrothaus et al., Eur. Phys. J. A 12, 147 (2001); H.V. Klapdor-Kleingrothaus, I.V. Krivosheina, A. Dietz, and O. Chkvorets, Phys. Lett. B 586, 198 (2004); C. Arnaboldi et al. (CUORICINO Collaboration), Phys. Rev. C 78 (2008) 035502; J. Wolf (KATRIN Collaboration), arXiv:0810.3281.

[21] C. Aalseth et al., arXiv:hep-ph/0412300; I. Abt et al., arXiv:hep-ex/0404039; Marco Vignati, EPJ Web Conf. 70 (2014) 00044

[22] J.A. Harvey and M.S. Turner, Phys. Rev. D 42 (1990) 3344.

[23] A. Abada, S. Davidson, F. X. Josse-Michaux, M. Losada and A. Riotto, JCAP 0604, (2006) 004 [arXiv:hepph/0601083]; S. Antusch, S. F. King and A. Riotto, JCAP 0611 (2006) 011 [arXiv:hep-ph/0609038].

[24] J. Dunkley et al., Astrophys. J. Suppl. 180 (2009) 306 [arXiv:astro-ph/0803.0593]. 